\title{
Processus de transport à travers la barrière hémato-encéphalique
}

\author{
J.M. Lefauconnier
}

INSERM U 26, hôpital Fernand Widal, 200, rue du Faubourg-Saint-Denis, 75475 Paris Cedex 10, France

(14 ${ }^{\mathrm{e}}$ Réunion du groupe Développement INRA, Clermont-Ferrand, 25-27 mai 1988)

Résumé - La barrière hémato-encéphalique est constituée par l'endothélium capillaire cérébral dont toutes les cellules sont liées aux cellules voisines par des jonctions intercellulaires étanches. Les processus de transport à travers cette barrière se font donc au niveau des 2 membranes de cette cellule endothéliale. La membrane luminale permet le passage des métabolites nécessalres au cerveau. La membrane abluminale joue un rôle important dans la sortie de substances du cerveau, sortie qui doit se faire dans un certain nombre de cas contre un gradient de concentration et nécessite donc la fourniture d'énergie. Les ions ont un transport très différent au niveau de ces 2 membranes. Le transport de sodium, de chlore est faible mais nécessite des transporteurs; le transport de potassium est très faible au niveau de la membrane luminale mais est important au niveau de la membrane abluminale, où il se fait par l'ATPase sodium-potassium dépendante. L'influx de glucose est très actif et se fait par transport facilité au niveau des membranes. L'efflux semble se faire par les mêmes transporteurs que l'influx. Les corps cétoniques passent du sang vers le cerveau et leurs transporteurs semblent mis en jeu pour la sortie d'acide pyruvique et surtout d'acide lactique. Les acides aminés sont transportés de façon très différente au niveau des 2 membranes. Au niveau de la membrane luminale siègent 2 transporteurs, celui des acides aminés basiques et celui des acides aminés neutres transportés par le système $L$. Au niveau de la membrane abluminale sont transportés tous les acides aminés neutres, en particulier ceux qui sont synthétisés dans le cerveau. Ces acides aminés sont transportés par le système L, le système A et le système ASC. Sont transportés également les acides aminés basiques et les acides aminés acides. Finalement, de nombreux autres processus de transport ont été identifiés au niveau de la barrière hémato-encéphalique pour les hormones, les vitamines et surtout les peptides. Au total, il semble que la barrière hématoencéphalique $a$ un rôle important à jouer pour la régulation de métabolisme cérébral.

cellule endothéliale du capillaire cérébral - transport de glucose - transport d'ions - transport d'acides monocarboxyliques - transport d'acides aminés — transport de peptides

Summary - Transport processes through the blood-brain barrier. The existence of the bloodbrain barrier is due to tight junctions between endothelial cells preventing the passage of liquid and solute material at the capillary level. Substances can thus pass across the blood-brain barrier if they are lipophilic or if they have transport systems in the membranes of endothelial cells. The luminal membrane brings metabolites needed for the brain function, the abluminal one plays an important part in removing substances from brain, this can happen against a concentration gradient and thus needs energy. lons are transported differently by the 2 membranes. Sodium and chloride have carriers and potassium is transported very actively by the sodium-potassium ATPase of the abluminal 
membrane. Blood-brain glucose influx is very important and happens by carrier transport at the 2 membranes. Efflux seems to use the same transport system as the influx. Transport of ketone bodies seems to happen only from blood to brain, the carriers being reversibly used for brain-blood transport of pyruvic and lactic acid. Amino-acid transport is very different on the luminal and abluminal membranes. On the luminal membrane there are 2 transport systems, one for basic amino acids, the other one, the $L$ system, for neutral amino-acids. All neutral amino-acids are transported through the abluminal membrane by the $L, A$ and ASC systems. There exists a system of transport for basic amino-acids, and a very active one for acid amino-acids. Some systems for the transport of hormones, vitamins and for some peptides exist also at the blood-brain barrier which thus plays a very important role in the regulation of brain metabolism.

brain capillary endothelium - glucose transport - ion transport - monocarboxylic acid transport - amino-acid transport - peptide transport

\section{INTRODUCTION}

La barrière hémato-encéphalique (BHE) est située à l'interface sang-espace extracellulaire cérébral. Elle est localisée au niveau des cellules endothéliales des capillaires cérébraux et de leurs jonctions (Reese \& Karnovsky, 1967; Brightman \& Reese, 1969). Les caractéristiques de l'endothélium capillaire cérébral : existence de jonctions cellulaires étanches, absence de fenestrations, très faible transfert vésiculaire et pinocytose, le font se comporter comme une membrane continue, ou plutôt comme un couche cellulaire épithéliale (Fenstermacher \& Rapoport, 1984; Betz, 1985; Crone, 1985). Cet endothélium contient par ailleurs un grand nombre de mitochondries (Oldendorf et al., 1977).

Pour passer du sang à l'espace extracellulaire cérébral, une substance doit donc franchir successivement la membrane luminale, le cytoplasme et la membrane abluminale de la cellule endothéliale. La traversée du cytoplasme est une barrière supplémentaire pour certaines molécules, dont les neurotransmetteurs, qui peuvent être dégradées par des enzymes cytoplasmiques (Hardebo \& Owman, 1980). Suivant la nature de la molécule étudiée, le transport à travers une membrane peut se faire : 1) à travers la double couche lipidique suivant un processus de diffusion simple. La vitesse d'influx est alors proportionnelle au gradient de concentration de part et d'autre de la membrane. Le coefficient de proportionnalité (coefficient de perméabilité surface) est le produit de la surface capillaire (S) et d'un coefficient $(P)$ qui dépend de la lipophilie de la molécule, 2) à l'aide d'un transporteur protéique. Les molécules hydrophiles que sont la plupart des substrats métaboliques ne peuvent traverser les membranes que grâce à une interaction réversible avec des protéines membranaires avec lesquelles elles possèdent une certaine complémentarité de structure. Si le transport se fait sans apport d'énergie, il est appelé diffusion facilitée et se fait dans le sens du gradient de concentration. Si le transport nécessite de l'énergie, il est appelé transport actif et peut se faire contre le gradient de concentration. Ce transport, par transporteur protéique, est caractérisé par sa stéréospécificité, sa saturabilité et une courbe de vitesse de transport en fonction de la concentration en substance transportée ressemblant à une courbe de réaction enzymatique. Nous n'envisagerons dans cet exposé que le transport par un transporteur protéique. II existe actuellement de plus en plus d'indications pour penser que les membranes luminales et 
abluminales sont différentes. Betz et al. (1980) ont en effet montré que la répartition des enzymes et des transporteurs variait d'une membrane à l'autre. La cellule endothéliale des capillaires cérébraux est donc polarisée et ressemble ainsi aux cellules épithéliales des plexus choroïdes, de l'intestin et du rein, ce qui explique en partie la comparaison du capillaire cérébral avec un épithélium serré. Ces caractéristiques seraient peut être induites par les astrocytes (Stewart \& Wiley, 1981; Janzer \& Raff, 1987).

Une autre barrière mérite d'être citée car elle peut contribuer à certains processus de transport, c'est la barrière hémoméningée. Cette barrière est située au niveau des plexus choroïdes, où il n'y a plus de jonctions étanches au niveau des cellules endothéliales du capillaire. Les jonctions étanches sont situées entre les cellules épithéliales du plexus, mais elles sont beaucoup plus perméables que celles du capillaire cérébral.

\section{MÉTHODES D'ÉTUDE}

Le transport d'une substance à travers la $\mathrm{BHE}$ est caractérisé par 3 vitesses : les vitesses d'influx, d'efflux et de transport net. Le transport net, différence entre l'influx et l'efflux, correspond à la vitesse d'incorporation de la substance dans le métabolisme cérébral.

\section{Mesures d'influx}

Elles mesurent la pénétration dans le cerveau de composés radioactifs injectés chez l'animal vivant soit par voie carotidienne, soit par voie veineuse (revue: Smith, 1985).

\section{Mesures d'efflux}

Elles nécessitent une injection par voie intracérébrale et il est souvent impossible de distinguer dans le résultat final le siège de l'efflux : capillaires cérébraux, plexus choroïdes, diffusion dans le liquide céphalorachidien (LCR) drainé dans les villosités arachnoïdiennes. Cependant une technique dont l'utilisation s'est développée donne des résultats intéressants : l'étude sur capillaires cérébraux isolés (pour une revue, voir Joó, 1985). Des études sur cultures de cellules endothéliales de capillaires cérébraux sont également utilisées (Bowman et al., 1983).

\section{TRANSPORT DES IONS}

Le transport hémato-encéphalique des ions est très lent comparé à celui des autres substrats traversant les capillaires (Bradbury, 1979; Fenstermacher \& Rapoport, 1984). La régulation de ce transport est capitale car elle maintient dans l'espace extra-cellulaire cérébral des concentrations ioniques stables et différentes de celles du plasma sanguin, ce qui permet le fonctionnement normal du système nerveux.

\section{Transport du sang vers le cerveau}

Les recherches faites récemment in vivo (Smith \& Rapoport, 1984, 1986) ont mis en évidence les difficultés d'étude de ce transport. En effet, l'autoradiographie après injection intraveineuse de ${ }^{36} \mathrm{Cl}$ a montré que la densité optique de la fixation du chlore radioactif était élevée dans les zones situées près des ventricules, ce qui traduit 
probablement l'association, dans ces zones, d'un influx provenant du plasma et du LCR, car les ions pénètrent beaucoup plus rapidement dans le LCR que dans le cerveau et diffusent ensuite dans le tissu cérébral.

Cette capture est diminuée par l'acétazolamide qui inhibe la formation de LCR. Cependant les mesures de radioactivité dans une zone éloignée des ventricules (cortex frontal) et pendant un temps assez court (10 min) ont permis de déterminer le transport transcapillaire et de montrer qu'il était encore plus faible que celui qui était obtenu par les mesures antérieures. Ces études ont également suggéré qu'une fraction significative du transport ionique se faisait par voie transcellulaire. Par ailleurs il a été montré que le transport de chlore était saturable (Smith \& Rapoport, 1984), ainsi que le transport de sodium (Betz, 1983). Ces 2 substances ont donc des transporteurs membranaires. Le transport de calcium est lui aussi fortement restreint mais il semble ne se faire que par diffusion simple (Tai et al., 1986).

\section{Transport du cerveau vers le sang}

Il est connu depuis un certain temps que le transport du potassium du cerveau vers le sang est plus important que le transport inverse du sang vers le cerveau. Bradbury \& Stulcova (1970) ont montré que l'efflux de potassium du cerveau se faisait par un système de transport dépendant de la concentration et inhibé par l'ouabaïne ce qui suggérait l'intervention dans ce transport de l'ATPase $\mathrm{Na}^{+}, \mathrm{K}^{+}$-dépendante. Sur les capillaires isolés, cette ATPase a été l'une des premières enzymes mises en évidence (Goldstein, 1979; Eisenberg \& Suddith, 1979). L'activité de cette enzyme dans les capillaires était élevée ce qui contraste avec un transport très faible in vivo. On a donc supposé qu'elle était localisée sur la membrane abluminale, ce qui a été confirmé par une étude cytochimique (Betz et al., 1980).

\section{TRANSPORT DE GLUCOSE}

Le glucose doit parvenir en quantité importante au cerveau afin d'alimenter le métabolisme énergétique cérébral dont il est le substrat presque exclusif chez l'adulte dans les conditions physiologiques.

\section{Description du système de transport}

En mesurant l'extraction de glucose à partir du glucose radioactif injecté dans la carotide, Crone (1965) a montré que celle-ci diminuait lorsque l'on augmentait la quantité de glucose dans le liquide injecté. $\mathrm{Ce}$ transport était donc saturable, ce qui le différenciait du transport de glucose à travers les capillaires des autres organes. Ces résultats ont été confirmés et précisés par des recherches faites in vivo (pour une revue, voir Lund-Andersen, 1979). Sur capillaires isolés, les études les plus récentes donnent des résultats concordant avec ceux qui ont été obtenus in vivo (Kolber et al., 1979).

Le transport transcapillaire de glucose est : a) saturable, b) stéréospécifique puisque le D-glucose, le D-mannose, le Dgalactose ainsi que certains analogues structuraux non physiologiques (2 désoxyglucose, 3 méthylglucose) sont transportés alors que des oses très voisins, le Lglucose et le fructose ne le sont pas, c) inhibé par la cytochalasine $\mathrm{B}$, la phlorétine 
et, à un degré moindre par la phlorizine, d) indépendant du sodium et non inhibé par l'ouabaïne et les inhibiteurs métaboliques, e) non modifié par l'insuline. Ce transport n'est donc pas un transport actif, comme dans les cellules intestinales, mais il se fait par diffusion facilitée, comme dans les érythrocytes où son effet est d'équilibrer les concentrations de part et d'autre de la membrane. La mesure des constantes cinétiques de transport, $K_{m}$ et $V_{m}$ a donné des résultats variables d'un laboratoire à l'autre pour des raisons méthodologiques (pour une revue, voir Lund-Andersen, 1979). Cependant, chez l'animal anesthésié il semble probable que le $K_{m}$ soit de 5 à $9 \mathrm{mM}$, c'est-à-dire proche de la concentration plasmatique en glucose et le $\mathrm{V}_{\mathrm{m}}$ d'environ $1,5 \mu \mathrm{mol} / \mathrm{min} / \mathrm{g}$.

\section{Modifications du transport}

Dans les conditions physiologiques, l'influx de glucose, environ 1 à $1,5 \mu \mathrm{mol} / \mathrm{min} / \mathrm{g}$ est quantitativement important par rapport à la concentration cérébrale qui est environ de $2 \mu \mathrm{mol} / \mathrm{g}$. Ceci entraîne un renouvellement très rapide du glucose cérébral. Cremer et al. (1981) ont en effet calculé que $65 \%$ environ du glucose était renouvelé par minute. Ce chiffre explique la mauvaise tolérance cérébrale à l'hypoglycémie.

La vitesse du transport net de glucose : $0,3 \mu \mathrm{mol} / \mathrm{min} / \mathrm{g}$ (Kety, 1957) est inférieure à la vitesse d'influx. L'étape limitante de l'utilisation de glucose est donc probablement l'entrée dans le métabolisme, c'est-àdire la phosphorylation catalysée par I'hexokinase. Cette enzyme ayant un $\mathrm{K}_{\mathrm{m}}$ très bas $(40 \mu \mathrm{M})$ est saturée à la concentration cérébrale en glucose $(2 \mathrm{mmol} / \mathrm{L})$. $\|$ se produit physiologiquement un efflux correspondant aux deux tiers environ de l'influx; il se fait par diffusion facilitée, par un système de transport ayant les mêmes caractéristiques que le système d'influx (Kolber et al., 1979).

On sait, depuis les travaux de Sokoloff et al. (1977), qu'il existe une différence importante dans l'utilisation métabolique du glucose suivant les régions du cerveau et, à l'intérieur d'une même région, suivant l'état fonctionnel des cellules cérébrales. II a aussi été montré qu'il existait dans chaque zone un couplage entre l'activité fonctionnelle, le débit sanguin et l'utilisation de glucose (pour une revue, voir Sokoloff, 1981). Le problème se pose de savoir par quel mécanisme se fait l'augmentation de transport net, nécessaire pour répondre aux besoins métaboliques accrus dans les régions cérébrales où l'activité est augmentée. II peut se produire une diminution de l'efflux ou un accroissement de l'influx. Cependant, certaines augmentations importantes d'activité impliquent une utilisation métabolique supérieure à celle qui est fournie par diminution de l'efflux. L'augmentation du transport net, dans ces conditions, nécessite une variation parallèle de l'influx. Cremer et al. (1981) ont montré qu'il existe effectivement une augmentation de l'influx de glucose lorsque I'utilisation croît en réponse à une utilisation fonctionnelle accrue. Dans leurs expériences, le rapport entre influx et efflux était constant et voisin de $2 / 1$. De plus, Hawkins et al. (1983) ont montré qu'il existait une différence d'influx d'une zone cérébrale à l'autre et que, dans les différentes zones, il existait une forte corrélation entre influx et consommation de glucose. Quel est alors le mécanisme de couplage entre débit sanguin cérébral, métabolisme énergétique et influx de glucose ? Une hypothèse a été émise à plusieurs reprises : l'augmentation de l'activité fonctionnelle entraînerait une augmentation du nombre de capillaires perfusés (Bolwig et al., 1977; Lund-Andersen, 1979; Gjedde, 1983; Haw- 
kins et al., 1983). Ce recrutement capillaire permettrait à la fois l'accroissement du débit sanguin cérébral et de l'influx de glucose par disponibilité supérieure des transporteurs. La différence de densité capillaire d'une région cérébrale à l'autre pourrait aussi rendre compte de la différence d'influx suivant les régions (Hawkins et al., 1983).

En plus de ces modifications rapides en réponse à une élévation de l'activité fonctionnelle, il existe des modifications du transport de glucose qui sont probablement dues à une modification du nombre des transporteurs dans la paroi capillaire : a) lors du développement : Moore et al., (1971), Daniel et al. (1978), Cremer et al. (1976) ont montré qu'il existe une augmentation de la vitesse maximale de transport du glucose, ce qui traduit probablement une augmentation du nombre des transporteurs. Celle-ci est contemporaine d'une modification du métabolisme cérébral : passage d'une utilisation importante de corps cétoniques chez l'animal immature à l'utilisation presque exclusive de glucose chez l'animal mature, b) lors d'une hyperglycémie chronique. Gjedde \& Crone (1981) ont montré que chez des rats atteints d'hyperglycémie chronique, la vitesse maximale de transport de glucose à travers la BHE diminuait du tiers.

\section{TRANSPORT DES ACIDES MONOCAR- BOXYLIQUES}

On pensait initialement que les acides monocarboxyliques à courte chaîne avaient un processus de transport facilité dans les capillaires cérébraux (Oldendorf, 1973) et que les acides monocarboxyliques à chaîne plus longue pénétraient s'ils étaient libres dans le plasma sanguin, de façon prédominante par diffusion passive. Mais il a été montré que les acides à chaîne moyenne ou à chaîne longue avaient aussi dans certains cas un processus de transport facilité (Spector, 1988).

\section{Description du systeme de transport}

Un transport stéréospécifique d'acide pyruvique et lactique a d'abord été décrit ( $\mathrm{Ne}$ moto \& Sveringhaus, 1971). II a ensuite été montré qu'il était saturable (Daniel et al., 1972) et permettait le transport des acides acétique, L-lactique, propionique et butyrique (Oldendorf, 1973). Les corps cétoniques peuvent aussi être utilisés par le cerveau dans des circonstances physiologiques (allaitement, jeûne). Leur transport est saturable (Gjedde \& Crone, 1975) et stéréospécifique (Moore et al., 1976).

Le bilan du transport net, mesuré par différence artério-veineuse, d'acide pyruvique et lactique a été trouvé négatif (Daniel et al., 1972). Ceci indique que l'efflux est supérieur à l'influx et l'on peut supposer que le système de transport sert surtout à éliminer le lactate produit par le métabolisme cérébral. En revanche le bilan du transport des acides cétoniques montre que le transport net est voisin de l'influx (Daniel et al., 1972). II n'y aurait donc pas d'efflux. ces acides sont donc utilisés par le cerveau à une vitesse égale à leur transport qui est ainsi l'étape limitante de leur utilisation métabolique. II a été suggéré par Cremer et al. (1976) que le système pourrait fonctionner en échangeant une molécule de corps cétonique, transportée du plasma dans le cerveau, contre une molécule de lactate transportée en sens inverse.

II a récemment été montré que les acides alpha-cétoniques provenant du métabolisme des acides aminés auraient le 
même transporteur. En revanche, les acides alpha-cétoniques aromatiques ne seraient pas transportés (Steele, 1986).

\section{Modifications du transport}

Au cours du développement, l'activité du système diminue (Moore et al., 1976; Cremer et al., 1976, 1979). Cette diminution est contemporaine d'une modification du métabolisme énergétique cérébral, le combustible principal étant les corps cétoniques chez l'animal immature alors que c'est le glucose chez l'aciulte. Le transport des corps cétoniques augmente chez les adultes après un jeûne prolongé (Gjedde \& Crone, 1975). Après anastomose portocave expérimentale, ce transport diminue (Sarna et al., 1979).

\section{TRANSPORT DES ACIDES AMINÉS}

Le cerveau a besoin d'un apport continu d'acides aminés pour la synthèse protéi-

\section{INELUXX SANG-CERYEAUIN VIVO}

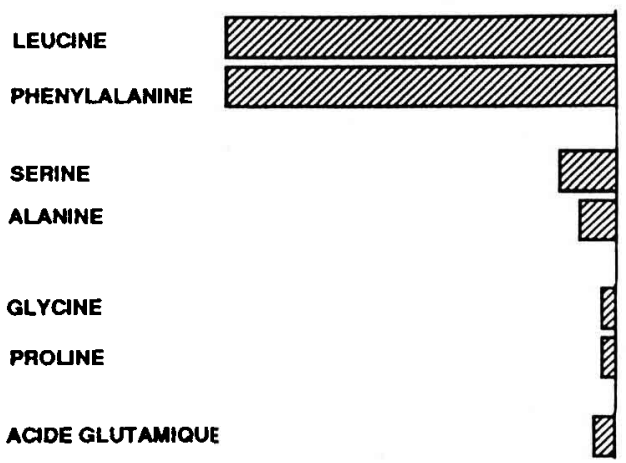

que, la synthèse de peptides, la synthèse de catécholamines et de sérotonine. La capacité de transport des membranes des cellules cérébrales est supérieure à celle de la $\mathrm{BHE}$, le transport à travers celle-ci est donc limitant pour la pénétration des acides aminés dans le cerveau.

\section{Description des systèmes de transport}

Le transport des acides aminés à travers les capillaires cérébraux est différent de celui des hexoses ou des acides monocarboxyliques. En effet, il comporte plusieurs systèmes de transport et il est asymétrique. Le passage à travers la membrane luminale du sang vers le cerveau est différent du passage inverse du cerveau vers le sang (Fig. 1).

\section{Transport transcapillaire du sang vers le cerveau}

Les premiers résultats d'Oldendorf (1971) ont montré que les acides aminés essen-

IRANSPORT DANS LES CAPILLAIRESISOLES

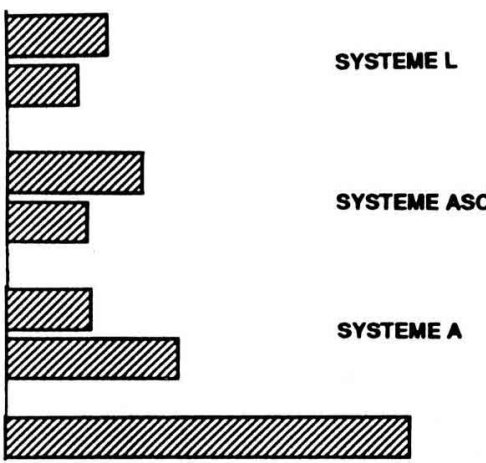

Fig. 1. Transport des acides aminés au niveau du capillaire cérébral chez le rat adulte. Sur cette figure sont indiqués, à gauche, les processus de transport in vivo. La longueur des rectangles est proportionnelle à l'index de capture cérébrale. A droite, est indiqué le transport des amino-acides mesuré sur capillaires isolés. La longueur des rectangles est proportionnelle à la radioactivité par $\mu \mathrm{g}$ de protéines. 
tiels sont mieux transportés que les acides aminés qui peuvent être synthétisés in situ. Les études de Richter \& Wainer (1971), Yudilevitch et al. (1972), Baños et al. (1973, 1974), Wade \& Katzman (1975) et Oldendorf \& Szabo (1976) ont montré qu'il existait 2 systèmes de transport des acides aminés du sang vers le cerveau. Un premier système transporte les grands acides aminés neutres et ressemble au système $L$ décrit par Christensen (1979), qui est sodium-indépendant et préfère les acides aminés avec chaîne latérale ou aromatiques. Un second système transporte les acides aminés basiques. Un troisième système décrit par Oldendorf et Szabo (1976) transporte les acides aminés acides et est peut-être seulement un système de transport plasma-cellule endothéliale. Sershen et Lajtha (1979) et Tovar et al. (1988) ont suggéré qu'il existait aussi un système de transport pour les petits acides aminés neutres (système ASC).

Le système de transport des grands acides aminés neutres (système $L$ ) est quantitativement le plus important; il transporte en particulier les précurseurs des neurotransmetteurs : tyrosine, tryptophane, DOPA. Pardridge \& Oldenforf (1975) ont montré que les $K_{m}$ des acides aminés transportés étaient voisins des concentrations plasmatiques, mais des études plus récentes (Smith et al., 1987) montrent que ces $\mathrm{K}_{\mathrm{m}}$ sont très inférieurs à ceux de la concentration plasmatique. Comme il n'existe qu'un seul transporteur pour un grand nombre d'acides aminés, des phénomènes de compétition se produisent. Pardridge (1977) a ainsi montré que l'influx cérébral d'un acide aminé n'est pas déterminé seulement par sa concentration plasmatique et son affinité pour le transporteur, mais aussi par la concentration plasmatique et l'affinité pour le transporteur des autres acides aminés neutres. Comme pour le glucose, il existe une im- portante différence d'influx suivant les zones cérébrales (Hawkins et al., 1982; Smith et al., 1985). Cette différence d'influx est peut-être due à la différence de densité capillaire.

\section{Transport transcapillaire du cerveau vers le sang}

Hjelle et al. (1978) ont montré que les capillaires isolés transportaient bien les grands acides aminés neutres. Betz et Goldstein (1978), d'autre part, ont observé que ces mêmes préparations transportaient l'acide $\alpha$-(méthylamino)isobutyrique, acide aminé synthétique caractéristique d'un système de transport sodiumdépendant des petits acides aminés neutres (système A). II existe donc dans les capillaires isolés un système de transport des petits acides aminés neutres, qui se trouve probablement situé sur la membrane abluminale puisque ce système n'existe pas in vivo et qui transporte les petits acides aminés du cerveau vers le sang, contre le gradient de concentration. Un système de transport ASC a également été montré dans les capillaires isolés (Tayarani et al., 1987) ainsi qu'un système très actif de transport de l'acide glutamique (Hutchinson et al., 1985).

\section{Modifications rapides du transport}

Ces modifications rapides résultent des phénomènes de compétition des acides aminés pour le transporteur. Comme le système de transport est saturé à $96 \%$ avec le groupe des grands acides aminés neutres, l'influx total de ces acides aminés dans le cerveau est indépendant des fluctuations normales des taux plasmatiques. Car si celles-ci montrent des variations 
quotidiennes, liées à la consommation d'aliments, elles se font, dans la plupart des cas, par un accroissement ou une diminution de tous les acides aminés neutres (Smith et al., 1985). Cependant, si l'on choisit de modifier fortement les concentrations plasmatiques en acides aminés, par exemple en administrant un repas ne comportant que des hydrates de carbone et un repas comportant un taux variable de protéines, on obtient des concentrations cérébrales en acides aminés qui sont très différentes et sont en relation avec le rapport : acide aminé/total des acides aminés compétiteurs dans le plasma (Fernstrom \& Faller, 1978).

Par ailleurs, quand la concentration d'un ou plusieurs acides aminés s'élève fortement dans le plasma, l'influx de cet acide aminé va augmenter et celui des compétiteurs décroître. Ceci entraîne une modification de la concentration cérébrale en acide aminé.

\section{Modifications prolongées du transport}

\section{Au cours du développement}

Le transport des acides aminés se modifie fortement au cours du développement. Les grands acides aminés neutres sont mieux transportés chez l'animal immature que chez l'animal adulte (Baños et al., 1978; Sershen \& Lajtha, 1976; Nagashima et al., 1987). Les index de pénétration cérébrale des petits acides aminés neutres transportés par le système ASC sont très supérieurs chez le jeune à ce qu'ils sont chez l'animal adulte (Lefauconnier \& Trouvé, 1983); il en est de même de l'index de pénétration des acides aminés basiques (Cornford et al., 1982). II existe de plus chez l'animal immature un système de transport A qui disparaît totalement chez l'adulte (Nagashima et al., 1987).

\section{Dans certains états pathologiques}

L'anastomose porto-cave expérimentale est utilisée comme modèle animal d'encéphalopathie d'origine hépatique. L'élévation des concentrations cérébrales des acides aminés aromatiques serait due à une augmentation importante de l'activité du transporteur des acides aminés neutres (James et al., 1978). Ceci confirmé par de nombreux auteurs (pour une revue, voir Rossi-Fanelli et al., 1987).

Le transport des acides aminés est également perturbé dans l'hypothyroïdie (Daniel et al., 1975; Lefauconnier et al., 1985). Un déséquilibre du transport des acides aminés plasmatiques a aussi été observé dans les amino-acidémies (Pratt, 1982).

\section{TRANSPORT D'AUTRES PETITES MO- LÉCULES}

Un système de transport pour la choline a également été décrit (Diamond, 1971; Cornford et al., 1978), ainsi que 2 systèmes de transport pour les précurseurs des acides nucléiques, l'un transportant l'adénine et l'autre certains nucléosides puriques et pyrimidiques (Cornford \& Oldendorf, 1975). Le système de transport de l'adénosine a aussi été identifié sur les préparations de capillaires isolés (Wu \& Phillis, 1982; Stefanovich, 1983; Kalaria \& Harik, 1986). Un système de transport a également été décrit pour les hormones thyroïdiennes (Pardridge \& Mietus, 1980) et plusieurs systèmes de transport pour les vitamines : pyridoxine, acide pantothénique, biotine (Spector, 1978; Spector et al., 
1986, 1987), thiamine monophosphate (Patrini et al., 1988).

\section{TRANSPORT DES PEPTIDES}

On a longtemps considéré que ces substances ne passaient pas la BHE. En effet, elles ont un haut poids moléculaire, ne sont pas liposolubles et on ne connaissait pas de système de transport. Mais ces notions ont commencé à se modifier lorsque Van Houten \& Posner (1979) ont montré qu'il existait, in vivo, des récepteurs à l'insuline sur les vaisseaux cérébraux. Un récepteur à l'insuline a également été mis en évidence sur les microvaisseaux cérébraux isolés humains (Frank \& Pardridge, 1981). L'hypothèse a alors été faite que s'il existait un récepteur à l'insuline sur le capillaire cérébral, il pourrait faire partie d'un système de transport hémato-encéphalique. Ce modèle serait analogue au modèle de transcytose de Bar et al. (1983) pour la fourniture d'insuline à travers la barrière endothéliale des capillaires non cérébraux. On croit que le processus de transcytose comporte 3 étapes : 1) une endocytose par la membrane luminale, 2) une diffusion à travers le cytoplasme endothélial, 3) une exocytose par récepteur par la membrane abluminale. Ceci a été montré après des essais sur des capillaires humains (Pardridge et al., 1985) et par des études sur de jeunes lapins (Duffy et al., 1986) : le rapport $\left({ }^{125} \mathrm{l}\right)$ insuline $/\left({ }^{3} \mathrm{H}\right)$ albumine augmentait après la perfusion carotidienne des 2 substances marquées. Par ailleurs, la capture cérébrale d'insuline était complètement inhibée par de fortes concentrations d'insuline froide dans le liquide de perfusion. Le devenir de l'insuline marquée a été étudié par autoradiographie montrant que les grains étaient concentrés autour des capillaires cérébraux avec une distribution qui pouvait cependant s'étendre loin du capillaire. Mais cela n'était pas une preuve ássolue de la transcytose car on ne connaissait pas la nature chimique du produit radioactif qui avait franchi la paroi capillaire. Une étude par HPLC a donc été effectuée après la perfusion par l'insuline marquée et l'on a pu voir que le métabolite radioactif principal était de l'insuline marquée et qu'il était immunoprécipitable par des anticorps anti-insuline.

L'endocytose d'autres peptides a également été montrée (Pardridge, 1986) : celui du facteur insuline-like (Frank et al., 1986), celui de la transferrine (Pardridge et al., 1987), celui d'albumine cationisée (Kumagai et al., 1986), celui de la leucineencéphaline (Zlokovic et al., 1987). II a aussi été montré que l'on pouvait induire le transport d'un peptide pour lequel n'a pas été décrit de transporteur en le couplant avec l'albumine cationisée (Kumagai et al., 1987).

\section{CONCLUSION}

L'étude des processus de transport à travers la barrière hémato-encéphalique montre qu'ils sont variés et très sélectifs. Ils permettent la fourniture au cerveau des métabolites dont il a besoin, dans un espace extra-cellulaire cérébral dont les concentrations ioniques et en acides aminés sont très différentes de celles du plasma sanguin. 


\section{RÉFÉRENCES}

Baños G., Daniel P.M., Moorhouse S.R. \& Pratt O.E. (1973) The influx of amino acids into the brain of the rat in vivo : the essential compared with some non essential amino acids. Proc. $R$. Soc. London B. 183, 59-70

Baños G., Daniel P.M. \& Pratt O.E. (1974) Saturation of a shared mechanism which transports L-arginine and L-lysine into the brain of the living rat. J. Physiol. 236, 29-41

Baños G., Daniel P.M. \& Pratt O.E. (1978) The effect of age upon the entry of some amino acids into the brain and their incorporation into cerebral proteins. Develop. Med. Child Neurol. 20, 335-346

Bar R.S., Derose A., Sandra A., Peacock M.L. \& Owen W.G. (1983) Insulin binding to microvascular endothelium of intact heart : a kinetic and morphometric analysis. Am. J. Physiol. 244, E447

Betz A.L. (1983) Sodium transport from blood to brain : inhibition by furosemide and amiloride. $J$. Neurochem. 41, 1158-1164

Betz A.L. (1985) Epithelial properties of brain capillary endothelium. Fed. Proc. 44, 2614-2615

Betz A.L. \& Goldstein G.W. (1978) Polarity of the blood brain barrier : neutral amino acid transport into isolated brain capillaries., Science 202, 225-227

Betz A.L., Firth J.A. \& Goldstein G.W. (1980) Polarity of the blood brain barrier : distribution of enzymes between the luminal and antiluminal membranes of brain capillary endothelial cells. Brain Res. 192, 17-28

Bolwig T.G., Hertz M.D. \& Holm-Jensen J. (1977) Blood brain barrier permeability during eletroshock seizures in the rat. Eur. J. Clin. Invest. 7, 95-100

Bowman P.D., Ennis S.R., Rarey K.E., Betz A.L. \& Goldstein G.W. (1983) Brain microvessel endothelial cells in tissue culture : a model for study of blood brain barrier permeability. Ann. Neurol. 14, 396-402

Bradbury M. (1979) The concept of a Blood Brain Barrier. Wiley \& Sons

Bradbury M.W.B. \& Stulcova B. (1970) Efflux mechanism contributing to the stability of the potassium concentration in cerebrospinal fluid. $J$. Physiol. (London) 208, 415-430
Brightman M.W. \& Reese T.S. (1969) Junctions between intimately apposed cell membrane in the vertebrate brain. J. Cell Biol. 40, 648-677

Christensen H.N. (1979) Development in amino acid transport, illustrated for the blood brain barrier. Biochem. Pharmacol. 28, 1989-1992

Cornford E.M., Oldendort W.H. (1975) Independent blood brain barrier transport systems for nucleic precursors. Biochim. Biophys. Acta 394, 211-219

Cornford E.M., Braun L.D. \& Oldendorf W.H. (1978) Carrier-mediated transport of choline and certain choline analogs. J. Neurochem. 30, 299308

Cornford E.M., Braun L.D. \& Oldendorf W.H. (1982) Developmental modulations of blood brain barrier permeability as an indictor of changing nutritional requirements in the brain. Pediatr. Res. 16, 324-328

Cremer J.E., Braun L. \& Oldendorf W.H. (1976) Changes during development in transport processes of the blood brain barrier. Biochim. Biophys. Acta 448, 633-637

Cremer J.E., Cunningham V.J., Pardridge W.M., Braun L.D. \& Oldendorf W.H. (1979) Kinetics of blood brain barrier transport of pyruvate, lactate and glucose in suckling, weanling and adult rats. J. Neurochem. 33, 439-445

Cremer J.E., Ray D.E., Sarna G.S. \& Cunningham V.J. (1981) A study of the kinetic behaviour of glucose based on simultaneous estimates of influx and phosphorylation in brain regions of rats in different physiologic states. Brain Res. $221,331-342$

Crone C. (1965) Facilitated transfer of glucose from blood into brain tissue. J. Physiol. 181, 103-113

Crone C. (1985) The blood brain barrier : a modified tight epithelium. In : The Blood Brain Barrier in Health and Disease (Bradbury, Rumsby and Suckling, eds). Ellis Horwood Ltd, Chichester, pp. 17-40

Daniel P.M., Love E.R., Moorhouse S.R., Pratt O.E. \& Wilson P. (1972) The movement of ketone bodies, glucose, pyruvate and lactate between the blood and the brain of rats. J. Physiol. 274, 141-148

Daniel P.M., Love E.R. \& Pratt O.E. (1975) Hypothyroidism and amino acid entry into brain and muscle. Lancet 2, 872 
Daniel P.M., Love E.R. \& Pratt O.E. (1978) The effect of age upon the influx of glucose into the brain. J. Physiol. 274, 141-148

Diamond I. (1971) Choline metabolism in brain. The role of choline transport and the effects of phenobarbital. Arch. Neurol. 24, 333-339

Duffy K.R., Choi T.B. \& Pardridge W.M. (1986) In vivo evidence that brain insulin originates from blood. Clin. Res. 34, 57A

Eisenberg H.M. \& Suddtih R.L. (1979) Cerebral vessels have the capacity to transport sodium and potassium. Science 206, 1083-1085

Fenstermacher J.D. \& Rapoport S.I. (1984) Blood brain barrier. In : Handbook of Physiology. The Cardiovascular System. IV. American Physiological Society, 21, 969-1000

Fernstrom J.D. \& Faller D.V. (1978) Neutral amino acids in the brain : changes in response to food ingestion. J. Neurochem. 30, 1531-1538

Frank H.J.L. \& Padridge W.M. (1981) A direct in vitro demonstration of insulin binding to isolated brain microvessels. Diabetes 30,757

Frank H.J.L., Pardridge W.M., Morris W.L., Rosenfeld R.G. \& Choi T.B. (1986) Binding and internalization of insulin and insulin-like growth factors by isolated brain microvessels. Diabetes $35,654-661$

Gjedde A. (1983) Modulation of substrate transport to the brain. Acta Neurol. Scand. 67, 3-25

Gjedde A. \& Crone C. (1975) Induction processes in blood-brain transfer of ketone bodies during starvation. Am. J. Physiol. 229, 1165-1169

Gjedde A. \& Crone C. (1981) Blood-brain glucose transfer : repression in chronic hyperglycemia. Science 214, 456-457

Goldstein G.W. (1979) Relation of potassium transport of oxidative metabolism in isolated brain capillaries. J. Physiol. (London) 286, 185195

Hardebo J.E. \& Owman C. (1980) Barrier mechanisms for neurotransmitter monoamines and their precursors at the blood-brain barrier interface. Ann. Neurol. 8, 1-11

Hawkins R.A., Mans A.M. \& Biebuyck J.F. (1982) Amino acid supply to individual cerebral structures in awake and anesthetized rats. $A m$. J. Physiol. 242, E1-E11

Hawkins R.A., Mans A.M., Davis D.W., Hibbard L.S., Lu D.M. (1983) Glucose availability to individual cerebral structures is correlated to glucose metabolism. J. Neurochem. 40, 179-190
Hjelle J.T., Baird-Lambert J., Cardinale G., Spector S. \& Udenfriend S. (1978) Isolated microvessels : the blood-brain barrier in vitro. Proc. Natl. Acad. Sci. USA 75, 4544-4548

Hutchinson H.T., Eisenberg H.M. \& Haber B. (1985) High-affinity transport of glumate in rat brain microvessels. Exp. Neurol. 87, 260-269

James J.H., Escourrou J. \& Fisher J.E. (1978) Blood-brain neutral amino acid transport activity in increased after portocaval anastomosis. Science 200, 1395-1397

Janzer R.C. \& Raff M.C. (1987) Astrocytes induce blood-brain barrier properties in endothelial cells. Nature $325,253-257$

Joó F. (1985) Review : the blood-brain barrier in vitro : ten years of research on microvessels isolated from the brain. Neurochem. Int. 7, 1-25

Kalaria F.N. \& Harik S.I. (1986) Nucléoside transporter of cerebral microvessels and choroid plexus. J. Neurochem. 47, 1849-1856

Kety S.S. (1957) The general metabolism of the brain in vivo. In : Metabolism of the Nervous System (Richter D., ed.) Pergamon Press, London, pp. 221-237

Kolber A.R., Bagnelli C.R., Krigman M.R., Hayward J. \& Morell P. (1979) Transport of sugars into microvessels isolated from rat brain : a model for the blood-bain barrier. J. Neurochem. 33, 419-432

Kumagai A.K., Eisenberg J. \& Pardridge W.M. (1987) Absorptive-mediated endocytosis of cationized albumin and a $\beta$-endorphin-cationized albumin chimeric peptide by isolated brain capillaries. J. Biol. Chem. 262, 15214-15219

Lefauconnier J.M. \& Trouvé R. (1983) Developmental changes in the pattern of amino acid transport at the blood-brain barrier in rats. Brain Res. 6, 175-182

Lefauconnier J.M., Lacombe P. \& Bernard G. (1985) Cerebral blood flow and blood-brain influx of some neutral amino acids in control and hypothyroid 16-day-old rat. J. Cereb. Blood Flow Metab. 5, 318-326

Lund-Andersen H. (1979) Transport of glucose from blood to brain. Physiol. Rev. 59, 305-352

Moore T.J., Lione A.P., Regen D.M., Tarpley H.L. \& Raines P.L. (1971) Brain glucose metabolism in the newborn rat. Am. J. Physiol. 221, 1746-1753

Moore T.J., Lione A.P., Sugden M.C. \& Regen $M$. (1976) Hydroxybutyrate transport in rat brain 
developmental and dietary modulations. Am. J. Physiol. 221, 1746-1753

Nagashima T., Lefauconnier J.M. \& Smith Q.R. (1987) Developmental changes in neutral amino acid transport accross the blood-brain barrier. $J$. Cereb. Blood Flow Metab. 7, (S1), S 524

Nemoto E.M. \& Sveringhaus J.W. (1971) The stereospecific influx permeability of rat bloodbrain barrier (BBB) to lactic acid (LA). Clin. Res. 19,146

Oldendorf W.H. (1971) Brain uptake of radiolobeled amino acids, amines and hexoses after arterial injection. Am. J. Physiol. 221, 1629-1639

Oldendorf W.H. (1973) Carrier-mediated bloodbrain barrier transport of short chain monocarboxylic organic acids. Am J. Physiol. 224, 14501453

Oldendorf W.H. \& Szabo J. (1976) Amino acid assignment to one of three blood-brain barrier arnino acid carriers. Am. J. Physiol. 230, 94-98

Oldendorf W.H., Cornford M.E. \& Brown W.J. (1977) The large apparent work capability of the blood-brain barrier : a study of the mitochondrial content of capillary endothelial cells in brain and other tissues of the rat. Ann. Neurol. 1, 409-417

Pardridge W.M. (1977) Kinetics of competitive inhibition of neutral amino acid transport across the blood-brain barrier. J. Neurochem. 28, 103108

Pardridge W.M. (1986) Receptor-mediated peptide transport through the blood-brain barrier. Endocr. Rev. 7, 314-330

Pardridge W.M. \& Oldendorf W.H. (1975) Kinetic analysis of blood-brain barrier transport of amino acids. Biochim. Biophys. Acta 401, 128136

Pardridge W.M. \& Mietus L.J. (1980) Transport of thyroid and steroid hormones through the blood-brain barrier of the newborn rabbit : primary role of protein bound hormone. Endocrinology 107, 1705-1710

Pardridge W.M., Eisenberg J. \& Yang J. (1985) Human blood-brain barrier insulin receptor. $J$. Neurochem. 44, 1771-1778

Pardridge W.M., Eisenberg J. \& Yang J. (1987) Human blood-brain barrier transferrin receptor. Metabolism Exp. 36, 892-895

Patrini C., Reggiani C., Laforenza U. \& Rindi G. (1988) Blood-brain transport of thiamine monophosphate in the rat : a kinetic study in vivo. $J$. Neurochem. 50, 90-93
Pratt O.E. (1982) Transport inhibition in the pathology of phenylketonuria and other inherited metabolic diseases. $J$. Inherited Metab. Dis. 5 (S2), 75-81

Reese T.S. \& Karnovsky M.J. (1967) Fine structural localization of a blood-brain barrier to exogenous peroxidase. J. Cell Biol. 34, 207-217

Richter J.J. \& Wainer A. (1971) Evidence for separate systems for the transport of neutral and basic amino acids across the blood-brain barrier. J. Neurochem. 18, 613-620

Rossi-Fanelli F., Cascino A., Strom R., CardelliCangiano P., Ceci F., Muscaritoli M. \& Cangiano C. (1987) Amino acids and hepatic encephalopathy. Progr. Neurobiol. 28, 277-301

Sarna G.S., Bradbury M.W.B., Cremer J.E., Lai J.C.K. \& Teal H.M. (1979) Brain metabolism and specific transport at the blood-brain barrier after portocaval anastomisis in the rat. Brain Res. $160,69-83$

Sershen H. \& Lajtha A. (1976) Capillary transport of amino acid in the developing brain. Exp. Neurol. 53, 465-474

Sershen H. \& Lajtha A. (1979) Inhibition pattern by analogs indicates the presence of ten or more transport systems for amino acids in brain cells. J. Neurochem. 32, 719-726

Smith Q.R. (1985) Methods to determine bloodbrain barrier permeability and transport. In : Neuromethods (A.A. Boulton, G.B. Burker, eds). Humana Press 1, 389-418

Smith Q.R. \& Rapoport S.I. (1984) Carriermediated transport of chloride across the bloodbrain barrier. $J$. Neurochem. 42, 754-763

Smith Q.R. \& Rapoport S.I. (1986) Cerebrovascular permeability coefficients to sodium, potassium and chloride. J. Neurochem. 46, 17321742

Smith Q.R., Takasato Y., Sweeney D.J. \& Rapoport S.I. (1985) Regional cerebrovascular transport of leucine as measured by the in situ brain perfusion technique. J. Cereb. Blood Flow Metab. 5, 300-311

Smith Q.R., Momma S., Aoyagi M. \& Rapoport S.I. (1987) Kenetics of neutral amino acid transport across the blood-brain barrier. J. Neurochem. 49, 1651-1658

Sokoloff L. (1981) Relationships among local functional activity, energy metabolism and blood flow in the central nervous system. Fed. Proc. $40,2311-2316$ 
Sokoloff L., Reivich M., Des Rosiers M.H., Patlak C.S., Pettigrew K.D., Sakurada O. \& Shinohara M. (1977) The $\left({ }^{14} \mathrm{C}\right)$ deoxyglucose method for the measurement of local cerebral glucose utilization : theory, procedure and normal value in the conscious and anesthetized albino rat. $J$. Neurochem. 28, 897-916

Spector R. (1978) Vitamin B6 transport in the central nervous system. In vivo studies. J. Neurochem. 30, 881-887

Spector R. (1988) Fatty acid transport through the blood-brain barrier. J. Neurochem. 50, 639643

Spector R. \& Mock D. (1987) Biotin transport through the blood-brain barrier. J. Neurochem. 48, 400-404

Spector R., Sivesind C. \& Kinzenbaw (1986) Pantothenic acid transport through the bloodbrain barrier. J. Neurochem. 47, 966-971

Stefanovich V. (1983) Uptake of adenosine by isolated bovine cortex microvessels. Neurochem. Res. 8, 1459-1469

Steele R.D. (1986) Blood-brain barrier transport of the $\alpha$-ketoacid analogs of amino acids. Fed. Proc. 45, 2060-2064

Stewart P.A. \& Wiley M.J. (1981) Developing nervous tissue induces formation of blood-brain barrier characteristics in invading endothelial cells : a study using quail-chick transplantation chimeras. Develop. Biol. 84, 183-192

Tai C.-Y., Smith Q.R. \& Rapoport S.I. (1986) Calcium influxes into brain and cerebrospinal fluid are linearly related to plasma ionized serum. Brain Res. 385, 227-236

Tayarani I., Lefauconnier J.M., Roux F. \& Bourre J.M. (1987) Evidence for an alanine, serine and cysteine system of transport in isolated brain capillaries. J. Cereb. Blood Flow Metab. 7, 585-591

Tovar A., Tews J.K., Torres N. \& Harper A.E. (1988) Some characteristics of threonine transport across the blood-brain barrier of the rat. $J$. Neurochem. 51, 1285-1293

Van Houten M. \& Posner B.l. (1979) Insulin binds to brain blood vessels in vivo. Nature 282, 623

Wade L.A. \& Katzman R. (1975) Synthetic amino acids and the nature of L DOPA transport at the blood-brain barrier. J. Neurochem. 25, 837842

Wu P.H. \& Phillis J.W. (1982) Uptake of adenosine by isolated rat brain capillaries. $J$. Neurochem. 38, 687-690

Yudilevitch D.L., De Rose N. \& Sepulveda F.U. (1972) Facilitated transport of amino acids through the blood-brain barrier of the dog studied in a single capillary circulation. Brain Res. 44, 459-478

Zlokovic B.V., Lipovac M.N., Begey D.J., Davson H. \& Rakic L. (1987) Transport of leucineenkephalin across the blood-brain barrier in the perfused guinea-pig brain. J. Neurochem. 49, 310-315 